\title{
Probabilistic and Dynamic Molecule-Disease Interaction Modeling for Drug Discovery
}

\author{
Tianfan Fu \\ Georgia Institute of Technology \\ Atlanta, GA, USA
}

\author{
Cao Xiao \\ Amplitude \\ San Francisco, CA, USA
}

\author{
Cheng Qian \\ IQVIA \\ Cambridge, MA, USA
}

\author{
Lucas M. Glass \\ IQVIA \\ Cambridge, MA, USA
}

\author{
Jimeng Sun \\ University of Illinois \\ Urbana-Champaign \\ Urbana, IL, USA
}

\begin{abstract}
Drug discovery aims at finding promising drug molecules for treating target diseases. Existing computational drug discovery methods mainly depend on molecule databases, ignoring valuable data collected from clinical trials. In this work, we propose PRIME to leverage high-quality drug molecules and drug-disease relations in historical clinical trials to narrow down the molecular search space in drug discovery. PRIME also introduces time dependency constraints to model evolving drug-disease relations using a probabilistic deep learning model that can quantify model uncertainty. We evaluated PRIME against leading models on both de novo design and drug repurposing tasks. Results show that compared with the best baselines, PRIME achieves $25.9 \%$ relative improvement (i.e., reduction) in average hit-ranking on drug repurposing and $47.6 \%$ relative improvement in success rate on de novo design.
\end{abstract}

\section{CCS CONCEPTS}

- Computing methodologies $\rightarrow$ Neural networks.

\section{KEYWORDS}

Drug discovery, Probabilistic deep learning

\section{ACM Reference Format:}

Tianfan Fu, Cao Xiao, Cheng Qian, Lucas M. Glass, and Jimeng Sun. 2021. Probabilistic and Dynamic Molecule-Disease Interaction Modeling for Drug Discovery. In Proceedings of the 27th ACM SIGKDD Conference on Knowledge Discovery and Data Mining (KDD '21), August 14-18, 2021, Virtual Event, Singapore. ACM, New York, NY, USA, 11 pages. https://doi.org/10.1145/ 3447548.3467286

\section{INTRODUCTION}

Drug discovery is notoriously time and resource consuming due to the need for experimental search over large drug molecule space. Traditional approaches are often inefficient and costly. For example, for de novo design that creates new drug molecules, researchers need to test large molecule libraries to identify molecules with desirable

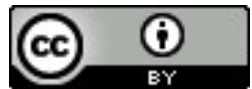

This work is licensed under a Creative Commons Attribution International 4.0 License. KDD '21, August 14-18, 2021, Virtual Event, Singapore.

(C) 2021 Copyright held by the owner/author(s). ACM ISBN 978-1-4503-8332-5/21/08.

https://doi.org/10.1145/3447548.3467286 properties [41, 61]. For drug repurposing that finds new indications for existing drugs, assay experiments need to be conducted to search over a large number of molecules in a candidate database [9, 23, 24].

The collection of massive biomedical data and the advances of deep learning technologies can potentially transform drug discovery tasks. Various deep learning models have recently shown encouraging performance in both de novo design and drug repurposing tasks. For de novo drug design, these models often use a large amount of druglike (or bioactive) molecules and leverage deep generative models or deep reinforcement learning to generate diverse and novel drug molecules to ultimately find molecules with desirable properties [17, 18, 20, 25, 26, 39, 47, 57, 59]. While drug repurposing is often done via modeling the interaction between existing drugs and protein targets [12, 31, 32, 38, 40, 44].

Despite these initial successes, existing works still face the following challenges.

- Restricted to limited labeled data. For de novo drug design, most of the existing methods mainly depend on a few molecule databases with limited labeled information about molecule docking or molecule properties [42, 43, 49]. For drug repurposing, current works often leverage limited knowledge in drug-disease or drug-protein target interactions to predict a large set of new potential indications.

- Lack of uncertainty estimation in prediction. Most existing works are based on maximum likelihood learning and cannot capture uncertainty in the prediction. However, even a model has good average performance for a set of predicted molecules; there are still out-of-distribution molecules of which the predictions are uncertain.

- Inadequate modeling of evolving molecule-disease relations. The relations of drug molecules and diseases evolve as our knowledge and experience with drugs grow [19, 46]. For example, Metamizole Sodium used to have a strong association with pain and fever (as a pain killer). Such association has been weakening due to a few side effects being discovered. In general, recent data often have more values than earlier ones. However, most existing methods are static, ignoring the evolving relations and importance of recent data.

To address these challenges, we leverage both drug discovery data and drug development data to extract high-quality molecule characteristics and evolving relations between drug molecules and 
diseases. We propose PRIME, a probabilistic and dynamic neural inference method to capture prediction uncertainty and drug-disease evolution with the following technical contributions.

- Augment the learning with high-quality clinical trial data. The molecules selected for development (e.g., clinical trials) are usually of high quality and exhibits better properties, and thus are often leveraged by human experts in drug design [22, 58]. In this work, we utilize these clinical trial data to model moleculedisease relations, narrow down the molecular space and improve the efficiency of both repurposing and de novo tasks.

- Probabilistic model to capture model uncertainty. We design the probabilistic and dynamic neural inference (PRIME) method to represent drug and disease as a high-dimensional distribution instead of regular point estimation. For repurposing tasks, such probabilistic modeling can select confident prediction and enhance the performance. For de novo design, uncertainty estimation allows thorough exploration to the chemical space.

- A dynamic neural inference module modeling evolving molecule-disease interactions. To imitate the evolving drugdisease relations, PRIME builds a dynamic model via imposing time dependency constraints on drug and disease representations in different time stamps.

- Theoretical analysis. We conduct theoretical analysis and reveal that PRIME can be seen as a weighting scheme under certain regularity conditions. This suggests PRIME can provide a flexible way to weigh earlier data and current data.

We evaluated PRIME on drug repurposing and de novo design tasks using real-world clinical trial data. Results show PRIME obtains $25.9 \%$ relative improvement compared with the best baseline method in hit-ranking on drug repurposing and enhance the success rate by $47.6 \%$ relative improvement on de novo drug design.

\section{RELATED WORK}

Probabilistic Deep Learning can capture uncertainty and avoids overfitting [6,53]. Meanwhile, dynamic models often exhibit superiority over static ones in the context of probabilistic models [51]. However, it is challenging to learn dynamic probabilistic neural networks due to the difficulty of optimizing time dependency. To address this issue, PRIME converts the time dependency constraint into parameter regularization in the learning objective and thus simplify the optimization process.

Deep Learning for Drug Discovery have shown initial success in drug repurposing and de novo design. For drug repurposing, models are usually based on either drug-target or drug-disease interaction $[13,32,38,40,44]$. Their models are trained with the static drugdisease/target database and did not consider the drug evolution and uncertainty estimation. This paper incorporates disease information from historical clinical trial data to help drug repurposing. For de novo drug design, existing works include deep generative model $[10,17,20,25,26]$, deep reinforcement learning [57], flow-based model [47, 59], or adversarial learning [11, 25, 39]. Most of these works focus on general-purpose de novo design, i.e., generating many novel and diverse molecules with desirable molecular properties and do not leverage the drug development knowledge. In contrast, PRIME focuses on disease-oriented de novo drug design, which designs drug molecule to cure target diseases. We use historical clinical trial records to narrow down the search scope and to model the evolution of drug-disease relations.

\section{METHOD}

\subsection{Problem Formulation}

Assume we have $k_{1}$ drugs in total, where the $i$-th drug is represented by its molecular structure $m_{i}$. The drug molecule set is $\mathcal{M}=\left\{m_{1}, m_{2}, \cdots, m_{k_{1}}\right\}$. Likewise, we assume there are $k_{2}$ disease codes in total. The disease code set is $\mathcal{D}=\left\{d_{1}, d_{2}, \cdots, d_{k_{2}}\right\}$. $\mathcal{T}=\{1,2, \cdots, t, \cdots, T\}$ is the set of $T$ continuous time stamps. Time stamp usually appears in superscript; for example, $\mathcal{M}^{(t)}$ represents the drug molecule set at time $t$. Then we define clinical trials and two drug discovery tasks below.

Definition 1 (Clinical Trial). A single clinical trial sample (denoted $x$ ) is registered at time stamp $\tau_{x} \in \mathcal{T}$ and test the drug molecule $m_{x} \in \mathcal{M}^{\left(\tau_{x}\right)}$ under the target disease set $D_{x} \subseteq \mathcal{D}$. The trial is denoted $x=\left[m_{x}, D_{x}, \tau_{x}\right]$.

Also, we use $\mathcal{X}^{(t)}$ to represent the set of all the trial samples at the time stamp $t$, use $\mathcal{X}^{\leq(t)}$ to represent the set of all the trial samples at and before the time stamp $t$.

Task 1 (Disease-oriented Drug Repurposing). At time $t$, for a new trial $x_{*}^{(t)}$ for the target disease set $D\left(x_{*}^{(t)}\right) \subseteq \mathcal{D}$, the goal is to find the appropriate drug molecule $m_{*} \in \mathcal{M}^{(t)}$ in the existing drug molecule set to conduct the trial.

Task 2 (Disease-oriented De Novo Drug Design). At time $t$, to design a new trial $x_{*}^{(t)}$ for the target disease set $D\left(x_{*}^{(t)}\right) \subseteq \mathcal{D}$, the goal is to design a novel molecule structure $m_{*}$ that does not exist in the current drug molecule set (i.e., $m_{*} \notin \mathcal{M}^{(t)}$ ) to treat the diseases.

\subsection{PRIME: probabilistic and dynamic neural inference for Molecule-Disease Interaction}

As illustrated in Fig. 1, PRIME first embeds drug molecules and diseases, then builds a dynamic probabilistic molecule-disease interaction model based on their embeddings. During the learning process, PRIME decomposes the complex objective function into a simple form using parameter regularization. The posterior of the prediction also supports uncertainty estimation to better guide decision making. Below we describe PRIME in details.

\section{A. Data Embedding of PRIME}

To prepare for molecule disease interaction modeling, we first embed both drug molecule and disease data as follows.

Molecule embedding: For each drug molecule $m$ represented as molecular graph, we use Molecular VAE (Variational Auto-Encoder) $[20,26,29,33,48]$ to represent and generate (reconstruct) $m$. Molecular VAE $f_{\phi}$ contains an encoder and a decoder. The encoder $f_{\phi}^{E}(\mathbf{h} \mid m)$ generates molecule representation $\mathbf{h}$ defined as in Eq. (1),

$$
\begin{aligned}
& \mathbf{h} \sim f_{\phi}^{E}(\mathbf{h} \mid m)=\mathcal{N}\left(\mu_{\phi}(m), \Sigma_{\phi}(m)\right), \quad \mathbf{h} \in \mathbb{R}^{c}, \\
& \mu_{\phi}(m)=g_{1}(\operatorname{MPN}(m)), \quad \log \left(\operatorname{diag}\left(\Sigma_{\phi}(m)\right)\right)=g_{2}(\operatorname{MPN}(m)) .
\end{aligned}
$$

Here $\phi$ is the parameter to learn. $\operatorname{diag}(Z) \in \mathbb{R}^{c}$ represents the diagonal of matrix $Z \in \mathbb{R}^{c \times c}$. MPN denotes the graph message-passing network that represents molecular graph $[16,26]$. It is followed by 


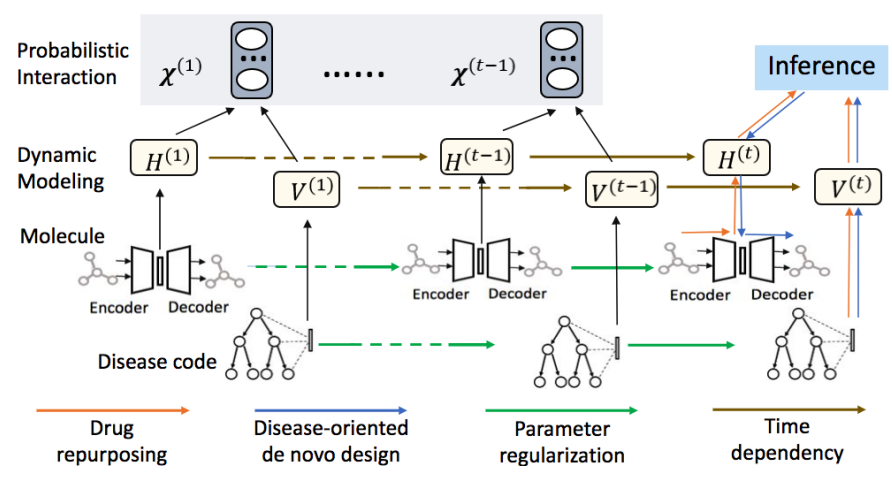

Figure 1: PRIME is a probabilistic neural model that models molecule-disease interaction dynamically. Drug molecules are embedded by VAE's encoder in Eq. (1), denoted H. Disease are embedded hierarchically in Eq. (4), denoted V. To impose the "time dependency" (Brown) for the dynamic model, we design the "parameter regularization" (Green) in Eq. (13). Given drug molecule and target disease, we conduct "drug repurposing” (Orange). For "disease-oriented de novo design", we are given target diseases, and infer the distribution of $h$ to generate new molecules based on decoder (Blue).

two separate fully-connected networks $g_{1}(\cdot), g_{2}(\cdot)$ that estimate $\mu_{\phi}$ and $\Sigma_{\phi}$, respectively. $\Sigma_{\phi}(m)$ is restricted to a diagonal matrix for computational tractability, a commonly-used approximation in Bayesian inference [60]. The decoder $f_{\phi}^{D}(m \mid \mathbf{h})$ reconstructs $m$ based on $\mathbf{h}$. The learning objective of VAE is given by Eq. (2),

$$
\mathcal{L}_{\mathrm{VAE}}=\mathrm{KL}\left(f_{\phi}^{E}(\mathbf{h} \mid m) \| p(\mathbf{h})\right)-\mathbb{E}_{f_{\phi}^{E}(\mathbf{h} \mid m)}\left[\log f_{\phi}^{D}(m \mid \mathbf{h})\right],
$$

where $p(\mathbf{h})$ is Gaussian with zero mean and unit variance. $\mathrm{KL}(\cdot \| \cdot)$ represents the KL divergence between two distributions. The first term of the loss encourages the variational distribution close to standard Gaussian distribution. The second term is the reconstruction loss for recovering the molecule $m$ based on $\mathbf{h}$.

Disease embedding: We take the ICD-10 disease codes [3] as input. They are hierarchically represented, where an ancestor represents a higher-level category of the current code. For example, in ICD 10 code [3], "D49" (neoplasm) and "D49.51" (kidney neoplasm) are the ancestors of "D49.511" (right kidney neoplasm). We follow previous works [15] to leverage the hierarchical information and represent disease $d_{j}$ as a linear combination of the basic embeddings ${ }^{1}\left(\mathbf{e} \in \mathbb{R}^{c}\right)$ of itself and its ancestors as in Eq. (3),

$$
\mathbf{w}_{j}=\sum_{k \in \mathcal{A}(j) \cup\{j\}} \alpha_{k j} \mathbf{e}_{k}, \quad \sum_{k} \alpha_{k j}=1,
$$

where $\alpha_{k j}$ represents normalized attention weight, $\mathcal{A}(j)$ represents the set of all the ancestors of $d_{j}$. To capture uncertainty, we suppose that disease representation $\mathbf{v}$ is drawn from a Gaussian distribution, whose mean and log-variance are parameterized as in Eq. (4),

$$
\begin{aligned}
& \mathbf{v}_{j} \sim g_{\eta}\left(d_{j}\right)=\mathcal{N}\left(\mu_{\eta}\left(d_{j}\right), \Sigma_{\eta}\left(d_{j}\right)\right), \quad \mathbf{v}_{j} \in \mathbb{R}^{c}, \\
& \mu_{\eta}\left(d_{j}\right)=g_{3}\left(\mathbf{w}_{j}\right) \in \mathbb{R}^{c}, \quad \log \left(\operatorname{diag}\left(\Sigma_{\eta}\left(d_{j}\right)\right)\right)=g_{4}\left(\mathbf{w}_{j}\right) \in \mathbb{R}^{c},
\end{aligned}
$$

${ }^{1}$ Co-occurrence information within a visit in electronic health record dataset is used to learn and initialize the basic embeddings of disease codes [15]. where $\eta$ is the parameter to learn, $g_{3}(\cdot), g_{4}(\cdot)$ are fully-connected networks, $\Sigma_{\eta}\left(d_{j}\right) \in \mathbb{R}^{c \times c}$ is restricted to a diagonal matrix for computational tractability.

In maximum likelihood estimation (MLE) methods, both $\mathbf{h}$ and $\mathbf{v}$ are embedding vectors parameterized directly by neural networks. Here, to quantify uncertainty, both $\mathbf{h}$ and $\mathbf{v}$ are drawn from Gaussian distributions whose mean and log-variance are parameterized by neural networks, as shown in Eq. (1) and (4). To obtain a warm start of representation, we pretrain molecule and disease representations on ZINC [49] (a large-scale molecule database) and MIMIC-III [27] (Electronic Health Record (EHR) dataset), respectively.

\section{B. Probabilistic Molecule-Disease Interaction Modeling}

The trial $x$ contains disease set $D_{x}$ and drug $m_{x}$. For each disease code $d \in D_{x}$, molecule-disease matching score is evaluated as $\sigma\left(\mathbf{h}_{m_{x}}^{\top} \mathbf{v}_{d}\right)$, where $\sigma(z)=\frac{1}{1+\exp (-z)}$ is the sigmoid function. The likelihood function is designed to be the geometric mean of all molecule-disease matching scores as in Eq. (5),

$$
p(x \mid \mathbf{H}, \mathbf{V})=\prod_{d \in D_{x}} \sigma\left(\mathbf{h}_{m_{x}}^{\top} \mathbf{v}_{d}\right)^{\frac{1}{\left|D_{x}\right|}},
$$

where $\mathbf{H}=\left\{\mathbf{h}_{1}, \cdots, \mathbf{h}_{k_{1}}\right\}$ denotes the set of all molecule embeddings, $\mathbf{V}=\left\{\mathbf{v}_{1}, \mathbf{v}_{2}, \cdots, \mathbf{v}_{k_{2}}\right\}$ denotes the set of all disease embeddings. Since all the available trials are regarded as positive samples, we need to provide noisy contrastive information. Thus we perform negative sampling following [37]. Specifically, we create a negative sample $x^{(-)}$for the trial $x$ as shown by Eq. (6),

$$
x^{(-)}=\left[m_{x}^{(-)}, D_{x}, \tau_{x}\right]
$$

where the disease information and time stamps are the same as the original trial $x$, and the original drug molecule $m_{x}$ will be replaced by $m_{x}^{(-)}$, according to two selection criteria: (1) $m_{x}^{(-)} \neq m_{x} ;(2)$ sampling probability is proportional to the drug's frequency in historical records, i.e., $m_{x}^{(-)} \sim \operatorname{Frequency}(m)$, for $m \in \mathcal{M}$. To provide contrastive information, we design the likelihood function of negative samples as in Eq. (7),

$$
\begin{aligned}
p^{\prime}\left(x^{(-)} \mid \mathbf{H}, \mathbf{V}\right) & =\prod_{d \in D_{x}}\left(1-\sigma\left(\mathbf{h}_{m_{x}^{(-)}}^{\top} \mathbf{v}_{d}\right)\right)^{\frac{1}{D_{x} \mid}} \\
& =\prod_{d \in D_{x}} \sigma\left(-\mathbf{h}_{m_{x}^{(-)}}^{\top} \mathbf{v}_{d}\right)^{\frac{1}{\left|D_{x}\right|}}
\end{aligned}
$$

where the second equation holds due to the property of sigmoid function. The negative to positive ratio is 1 , following $[53,56]$. That is, each positive sample corresponds to 1 negative sample.

To summarize, the joint likelihood function is given by Eq. (8),

$$
P(X \mid \mathbf{H}, \mathbf{V})=\prod_{\left(x, x^{(-)}\right) \in \mathcal{X}} p(x \mid \mathbf{H}, \mathbf{V}) p^{\prime}\left(x^{(-)} \mid \mathbf{H}, \mathbf{V}\right),
$$

where $X$ denotes the set of all trials including the negative samples.

\section{Capture Evolving Interactions}

The molecule-disease relations evolve as human's understanding of the drugs improves [19,46]. When designing a new clinical trial, recent clinical trials offer more values compared with old trials. We build a filtering model that only uses the past data during 
inference, which is consistent with the design of real-world trials. Thus, we construct the following dynamic model to capture the evolving relations. Specifically, there are $T$ continuous time stamps, $1,2, \cdots, T$, the trial set at time $t$ is denoted $\mathcal{X}^{(t)}$. we assume $\mathbf{H}$ and $\mathbf{V}$ evolve over time. At time $t$, we have $\mathbf{H}^{(t)}=\left\{\mathbf{h}_{1}^{(t)}, \mathbf{h}_{2}^{(t)}, \cdots\right\}$ and $\mathbf{V}^{(t)}=\left\{\mathbf{v}_{1}^{(t)}, \mathbf{v}_{2}^{(t)}, \cdots\right\}$.

Time Dependency refers to the relation between $\mathbf{h}^{(t)} / \mathbf{v}^{(t)}$ and earlier $\mathbf{h} / \mathbf{v s}$, i.e., $\mathbf{h}^{(1)}, \cdots, \mathbf{h}^{(t-1)} / \mathbf{v}^{(1)}, \cdots, \mathbf{v}^{(t-1)}$. To make it tractable, we assume both $\mathbf{h}$ and $\mathbf{v}$ follow rule of Markovian dynamical system [60]. That is, $\mathbf{h}^{(t)}$ depends only on $\mathbf{h}^{(t-1)}$ and $\mathbf{v}^{(t)}$ depends only on $\mathbf{v}^{(t-1)}$. Also, we leverage Brownian motion [51] to model their evolution over time. At time stamp $t$, the prior for $\mathbf{h}_{i}^{(t)}$ and $\mathbf{v}_{j}^{(t)}$ is an aggregation of posterior at previous time stamp $t-1$ and zero-mean Gaussian that imitates Brownian motion as in Eq. (9),

$$
\mathbf{h}_{i}^{(t)}\left|\mathbf{h}_{i}^{(t-1)} \sim \mathcal{N}\left(0, \sigma_{1}^{2} I\right), \quad \mathbf{v}_{j}^{(t)}\right| \mathbf{v}_{j}^{(t-1)} \sim \mathcal{N}\left(0, \sigma_{2}^{2} I\right),
$$

where $I \in \mathbb{R}^{c \times c}$ is the identity matrix. $\sigma_{1}^{2}, \sigma_{2}^{2}$ are hyperparameters that control the intensity of Brownian motion discussed in Section 4. The likelihood function at time $t$ is given below,

$$
p\left(\mathcal{X}^{(t)} \mid \mathbf{H}^{(t)}, V^{(t)}\right)=\prod_{\left(x, x^{(-)}\right) \in \mathcal{X}^{(t)}} p\left(x \mid \mathbf{H}^{(t)}, \mathbf{V}^{(t)}\right) p^{\prime}\left(x^{(-)} \mid \mathbf{H}^{(t)}, \mathbf{V}^{(t)}\right),
$$

where $x^{(-)}$is the negative sample of $x$ defined in Eq. (6). During the inference process, we compute the posterior distribution of $\mathbf{H}^{(t)}$ and $\mathbf{V}^{(t)}$ at time $t$ as in Eq. (10),

$$
p\left(\mathbf{H}^{(t)}, \mathbf{V}^{(t)} \mid \mathcal{X}^{\leq(t)}\right) \propto p\left(\mathbf{H}^{(t)}\right) p\left(\mathbf{V}^{(t)}\right) p\left(\mathcal{X}^{(t)} \mid \mathbf{H}^{(t)}, \mathbf{V}^{(t)}\right),
$$

where $\mathcal{X}^{\leq(t)}$ represents the set of trials at and before time $t$, the prior $p\left(\mathbf{H}^{(t)}\right)$ and $p\left(\mathbf{V}^{(t)}\right)$ at time $t$ depend on posterior at $\mathrm{t}-1$, as defined in Equation (9).

\section{Learning PRIME}

To describe the learning process of PRIME, we abuse the notation a bit for ease of exposition, use $\Theta$ to replace the parameter of interest $\Theta^{(t)}=\left\{\mathbf{H}^{(t)}, \mathbf{V}^{(t)}\right\}$ at time $t$, use $\Psi$ to replace the neural network parameters $\Psi^{(t)}=\left\{f_{\phi}^{(t)}, g_{\eta}^{(t)}\right\}$. At time $t$, we are interested in evaluating the posterior $p\left(\Theta \mid \mathcal{X}^{(t)}\right) \propto p(\Theta) p\left(\mathcal{X}^{(t)} \mid \Theta\right)$. In general cases, the posteriors do not own a closed-form solution and are mostly computationally intractable. To perform efficient inference, we leverage variational inference. The core idea of variational inference is to approximate Bayesian posterior with a simpler parameterized variational distribution. Specifically, $q\left(\Theta ; \mathcal{F}\left(\mathcal{X}^{(t)} ; \Psi\right)\right)$ is used to approximate $p\left(\Theta \mid \mathcal{X}^{(t)}\right), \mathcal{F}$ can be any neural network with parameter $\Psi$ and input $\mathcal{X}^{(t)} . \mathcal{F}\left(\mathcal{X}^{(t)} ; \Psi\right)$ is the variational parameter that characterizes variational distribution $q\left(\Theta ; \mathcal{F}\left(\mathcal{X}^{(t)} ; \Psi\right)\right)$. Then we minimize the KL divergence between the variational distribution and the true posterior. It is reformulated as an optimization problem and can be solved efficiently. Specifically, at time $t$, the objective is a function of neural networks' parameter $\Psi$ as in Eq. (11),

$$
\mathcal{L}(\Psi)=\mathrm{KL}\left(q\left(\Theta ; \mathcal{F}\left(\mathcal{X}^{(t)} ; \Psi\right)\right) \| p\left(\Theta \mid \mathcal{X}^{(t)}\right)\right),
$$

which is an integration. As shown in Equation (9), the current prior is an aggregation of the posterior at previous time and a zeromean Gaussian distribution, where the previous time's posterior is parameterized by the same neural architecture. It makes the prior complex and intractable. To address the issue, we simplify the objective as follows.

Lemma 1. The objective in Eq. (11) can be decomposed as below,

$$
\begin{aligned}
\mathcal{L}(\Psi) & =\underbrace{\operatorname{KL}\left(q\left(\Theta ; \mathcal{F}\left(\mathcal{X}^{(t)} ; \Psi\right)\right) \| p(\Theta)\right)}_{\mathcal{L}_{1}(\Psi)} \\
& -\underbrace{\mathbb{E}_{q\left(\Theta ; \mathcal{F}\left(\mathcal{X}^{(t)} ; \Psi\right)\right)}\left[\log p\left(\mathcal{X}^{(t)} \mid \Theta\right)\right]}_{\mathcal{L}_{2}(\Psi)}+\text { Constant, }
\end{aligned}
$$

where $p(\Theta)$ is the prior of $\Theta\left(\Theta^{(t)}\right)$. According to Eq. (9), the prior of $\Theta^{(t)}$ is related to the posterior of $\Theta^{(t-1)}, \mathcal{L}_{1}$ can be further simplified. We make the Lipschitz assumption on the logarithm of a variational density function.

Assumption 1 (Lipschitz Condition). The logarithm of variational density function $\log q\left(\Theta ; \mathcal{F}\left(\mathcal{X}^{(t)} ; \Psi\right)\right)$ is L-Lipschitz in $\Psi$ for any $\Theta$. That is, for $\forall \Theta, \Psi_{1}, \Psi_{2}$, we have

$$
\left|\log q\left(\Theta ; \mathcal{F}\left(\mathcal{X}^{(t)} ; \Psi_{1}\right)\right)-\log q\left(\Theta ; \mathcal{F}\left(\mathcal{X}^{(t)} ; \Psi_{2}\right)\right)\right| \leq L|| \Psi_{1}-\Psi_{2} \|,
$$

where $\|\cdot\|$ represent the $L_{2}$ norm. It is a common and mild assumption for variational distribution [35]. Then we provide an upper bound for $\mathcal{L}_{1}(\Psi)$ as follows.

Lemma 2. If Assumption 1 holds, $\mathcal{L}_{1}(\Psi)$ can be bounded as $\mathcal{L}_{1}(\Psi) \leq$ $L\left\|\Psi-\Psi^{(t-1)}\right\|$.

For computational convenience, we use $\left\|\Psi-\Psi^{(t-1)}\right\|^{2}$ as a surrogate of $\left\|\Psi-\Psi^{(t-1)}\right\|$. Now the learning objective becomes

$$
\begin{aligned}
\mathcal{L}(\Psi) & =-\mathbb{E}_{q\left(\Theta ; \mathcal{F}\left(\mathcal{X}^{(t)} ; \Psi\right)\right)}\left[\log p\left(\mathcal{X}^{(t)} \mid \Theta\right)\right]+\lambda\left\|\Psi-\Psi^{(t-1)}\right\|^{2} \\
& =\mathcal{L}_{\text {data }}(\Psi)+\mathcal{L}_{\text {regularization }}(\Psi) .
\end{aligned}
$$

where $\lambda>0$ is a hyperparameter. $\mathcal{L}_{\text {data }}(\Psi)$ is the expectation of log-likelihood for current data $\mathcal{X}^{(t)}$. parameter regularization $\mathcal{L}_{\text {regularization }}(\Psi)$ encourage the current model to be close to the model at the last time stamp, which encodes the knowledge learned from previous data $\mathcal{X}^{\leq(t-1)}$. The time dependency inherent in the prior $p\left(\Theta^{(t)}\right)$ is converted into the parameter regularization, as illustrated in Figure 1 . The gradient of $\mathcal{L}_{\text {data }}(\Psi)$ is evaluated as

$$
\begin{aligned}
& \nabla_{\Psi} \mathcal{L}_{\text {data }}(\Psi)=-\nabla_{\Psi} \mathbb{E}_{q\left(\Theta ; \mathcal{F}\left(\mathcal{X}^{(t)} ; \Psi\right)\right)}\left[\log p\left(\mathcal{X}^{(t)} \mid \Theta\right)\right] \\
= & -\int \nabla_{\Psi} q\left(\Theta ; \mathcal{F}\left(\mathcal{X}^{(t)} ; \Psi\right)\right) \log p\left(\mathcal{X}^{(t)} \mid \Theta\right) d \Theta \\
= & -\mathbb{E}_{q\left(\Theta ; \mathcal{F}\left(\mathcal{X}^{(t)} ; \Psi\right)\right)}\left[\nabla_{\Psi} \log q\left(\Theta ; \mathcal{F}\left(\mathcal{X}^{(t)} ; \Psi\right)\right) \log p\left(\mathcal{X}^{(t)} \mid \Theta\right)\right],
\end{aligned}
$$

where the third line uses log-derivative trick [60]. The gradient can be estimated by Monte Carlo samples from $q\left(\Theta ; \mathcal{F}\left(\mathcal{X}^{(t)} ; \Psi\right)\right)$.

Since $\Theta=\{\mathbf{H}, \mathbf{V}\}$ and $\Psi=\left\{f_{\phi}, g_{\eta}\right\}$, to make the inference process tractable, we make the mean-field assumption ${ }^{2}$ that posterior of

\footnotetext{
${ }^{2}$ Mean-field assumption is commonly used technique in variational inference, which assume that the unknown variables are independent of the others [60].
} 
$\mathrm{H}, \mathrm{V}$ can be fully factorized as

$$
\begin{aligned}
& p\left(\mathbf{H}^{(t)}, \mathbf{V}^{(t)} \mid X^{\leq(t)}\right) \approx q\left(\mathbf{H}^{(t)} ; f_{\phi}\right) q\left(\mathbf{V}^{(t)} ; g_{\eta}\right) \\
\approx & \prod_{\mathbf{h} \in \mathbf{H}^{(t)}} q\left(\mathbf{h} ; f_{\phi}\right) \prod_{\mathbf{v} \in \mathbf{V}^{(t)}} q\left(\mathbf{v} ; g_{\eta}\right) .
\end{aligned}
$$

Following the idea of Gibbs sampling [60], we alternatively optimize $\mathbf{H}$ (i.e., $f_{\phi}$ ) and $\mathbf{V}$ (i.e., $g_{\eta}$ ). Since different $\mathbf{h}_{i} / \mathbf{v}_{j}$ s are fully decomposable in posterior in Eq. (10), we can optimize different $\mathbf{h}_{i} / \mathbf{v}_{j} \mathrm{~s}$ in parallel. When optimizing H, we include VAE's objective (Eq. (2)) to finetune the VAE using high-quality drug molecules.

\section{E. Uncertainty Estimation}

Below we will discuss how to perform uncertainty quantification for the two drug discovery tasks. For both tasks, we make prediction at time $t$, which has the variational distribution $q\left(\mathbf{h}_{i}^{(t-1)}\right)$ and $q\left(\mathbf{v}_{j}^{(t-1)}\right)$ at previous time $\mathrm{t}-1$. For all these tasks, we are given the set of target diseases, $D_{x} \subseteq \mathcal{D}$.

Drug Repurposing. Given a repurposed drug molecule $m$, we are interested in the expectation of molecule-disease matching score under the posterior distributions $q\left(\mathbf{h}_{m}^{(t-1)}\right), q\left(\mathbf{v}_{d}^{(t-1)}\right)$ for $d \in D_{x}$, i.e.,

$$
\mathcal{S}^{(t)}\left(m, D_{x}\right)=\mathbb{E}\left[q\left(\mathbf{h}_{m}^{(t-1)}\right) \prod_{d \in D_{x}} q\left(\mathbf{v}_{d}\right)\right]\left[\prod_{d \in D_{x}}\left(\sigma\left(\mathbf{h}_{m}^{\top} \mathbf{v}_{d}\right)\right)^{\frac{1}{\left|D_{x}\right|}}\right]
$$

We use the Monte Carlo samples to estimate both mean and standard deviation of $\mathcal{S}^{(t)}\left(m, D_{x}\right)$, denoted $\mu_{S}(m)$ and $\sigma_{s}(m)$, respectively. Drug repurposing is equivalent to find the molecules with the highest matching score, i.e.,

$$
\underset{m \in \mathcal{M}^{(t-1)}}{\arg \max } \widehat{\mathcal{S}^{(t)}}\left(m, D_{x}\right), \quad \widehat{\mathcal{S}^{(t)}}\left(m, D_{x}\right)=\mu_{s}(m)-\gamma \sigma_{s}(m)
$$

where $\mathcal{M}^{(t-1)}$ is the existing drug molecule set at time $\mathrm{t}-1, \gamma>0$ is a hyperparameter and is set to 0.3 , following Bayesian inference tricks [8]. To select predictions with high confidence, we use $\mu_{s}(m)-$ $\gamma \sigma_{s}(m)$ to replace the mean $\mu_{s}(m)$. Predictions with high confidence have lower standard deviation and higher scores. Compared with $\mu_{s}(m)$, it does improve performance, as shown in Section 5.5.

Disease-oriented De Novo Drug Design. Different from repurposing, we do not have the molecule $m$ and the variational distribution $q\left(\mathbf{h}_{m}^{(t-1)}\right)$. Instead, we are interested in drawing $\mathbf{h}$ samples from the following distribution,

$$
Q(\mathbf{h})=\mathbb{E}_{\left[\prod_{d \in D_{x}} q\left(\mathbf{v}_{d}\right)\right]}\left[p(\mathbf{h}) \prod_{d \in D_{x}}\left(\sigma\left(\mathbf{h}^{\top} \mathbf{v}_{d}\right)\right)^{\frac{1}{\left|D_{x}\right|}}\right] .
$$

We can use Markov Chain Monte Carlo (MCMC) method to sample $\tilde{\mathbf{h}}^{(1)}, \tilde{\mathbf{h}}^{(2)}, \cdots$ from the distribution, then generate new molecules via feeding the sampled $\tilde{\mathbf{h}}^{(i)}$ into the decoder $f_{\phi}^{D}$ in VAE (Section 3.2 and Fig. 1). However, the posterior in Eq (17) is computationally intractable; standard MCMC cannot handle it. Instead, we use Stochastic Gradient Langevin Dynamics (SGLD) [52], which only requires the noisy gradient of the logarithm of posterior. The noisy gradient can be approximated as

$$
\nabla_{\mathbf{h}} \log Q(\mathbf{h}) \approx \nabla_{\mathbf{h}} \log p(\mathbf{h})+\frac{1}{K} \sum_{k=1}^{K} \frac{1}{\left|D_{x}\right|} \sum_{d \in D_{x}} \nabla_{\mathbf{h}} \log \sigma\left(\mathbf{h}^{\top} \mathbf{v}_{d}^{(k)}\right),
$$

where $\mathbf{v}_{d}^{(1)}, \cdots, \mathbf{v}_{d}^{(K)} \stackrel{\text { i.i.d. }}{\sim} \mathcal{N}\left(\mu_{v_{d}}, \Sigma_{v_{d}}\right)$ are $K$ Monte Carlo samples independent and identically distributed (i.i.d.) from the variational distribution. The SGLD sampling process is

$$
\tilde{\mathbf{h}}^{(l+1)}=\tilde{\mathbf{h}}^{(l)}+\epsilon_{l} \nabla_{\mathbf{h}} \log Q\left(\tilde{\mathbf{h}}^{(l)}\right)+\sqrt{\epsilon_{l}} \xi, \quad \xi \sim \mathcal{N}(0, I)
$$

where $\tilde{\mathbf{h}}^{(l)}$ is the sample at $l$-th step, $\epsilon_{l}$ is the stepsize, satisfying Robbins-Monro conditions [52], i.e., $\sum_{l=1}^{\infty} \epsilon_{l}=+\infty, \sum_{l=1}^{\infty} \epsilon_{l}^{2}<+\infty$. Under these conditions, it is guaranteed that the sampled $\tilde{\mathbf{h}}^{(l)} \mathrm{s}$ asymptotically converge to the target posterior [52].

\section{THEORETICAL ANALYSIS}

Next, we provide the theoretical analysis of dynamic modeling under some regularity conditions. Without loss of generality, we restrict our attention to the posterior of $\mathbf{h}_{i}^{(t)}$ and it can be generalized to $\mathbf{v}_{j}^{(t)}$ for any timestamp $t$ using the same analysis. First, we make the Bayesian asymptotic assumption and then present theoretical results and some discussion. The proofs are given in Supplementary Material.

Assumption 2 (Bayesian Central Limit Theorem [1]). When number of samples is large enough, the posterior asymptotically approaches to the normal distribution, i.e., $p\left(\mathbf{h}_{i}^{(t)} \mid \mathcal{X}^{\leq(t)}\right)=\mathcal{N}\left(\mu_{h_{i}}^{(t)}, \Sigma_{h_{i}}^{(t)}\right)$.

Lemma 3. Under Assumption 2, the likelihood function $p\left(X^{(t)} \mid \mathbf{h}_{i}^{(t)}\right)$ follows Gaussian distribution, whose mean and variance are denoted $\hat{\mu}_{h_{i}}^{(t)}$ and $\hat{\Sigma}_{h_{i}}^{(t)}$, respectively, i.e., $p\left(\mathcal{X}^{(t)} \mid \mathbf{h}_{i}^{(t)}\right)=\mathcal{N}\left(\hat{\mu}_{h_{i}}^{(t)}, \hat{\Sigma}_{h_{i}}^{(t)}\right)$.

Note that $\hat{\mu}_{h_{i}}^{(t)}$ and $\hat{\Sigma}_{h_{i}}^{(t)}$ are mean and variance for likelihood function while $\mu_{h_{i}}^{(t)}$ and $\Sigma_{h_{i}}^{(t)}$ corresponds to posterior. Then we decompose the posterior at time $t$.

Theorem 1. Under Assumption 2, the mean and variance of the posterior $\mathcal{N}\left(\mu_{h_{i}}^{(t)}, \Sigma_{h_{i}}^{(t)}\right)$ are

$\mu_{h_{i}}^{(t)}=\mathrm{S}\left(\left(\left(\Sigma_{h_{i}}^{(t-1)}\right)^{-1}+\frac{1}{\sigma_{1}^{2}} I\right) \mu_{h_{i}}^{(t-1)}+\left(\hat{\Sigma}_{h_{i}}^{(t)}\right)^{-1} \hat{\mu}_{h_{i}}^{(t)}\right), \quad \Sigma_{h_{i}}^{(t)}=\mathrm{S}$,

where $\mathrm{S}=\left(\left(\Sigma_{h_{i}}^{(t-1)}\right)^{-1}+\frac{1}{\sigma_{1}^{2}} I+\left(\hat{\Sigma}_{h_{i}}^{(t)}\right)^{-1}\right)^{-1}, \sigma_{1}^{2}$ is defined in Eq. (9). The hyperparameter $\sigma_{1}^{2}$ controls the importance of earlier data on the current model. Corollary of Theorem 1 shows the limit property of $\sigma_{1}^{2}$.

Corollary 1. Under Assumption 2, when $\sigma_{1}^{2}$ goes to positive infinity, the posterior $p\left(\mathbf{h}_{i}^{(t)} \mid X^{\leq(t)}\right)$ is approaching to

$$
\mu_{h_{i}}^{(t)}=\mathrm{S}\left(\sum_{\tau=1}^{t}\left(\hat{\Sigma}_{h_{i}}^{(\tau)}\right)^{-1} \hat{\mu}_{h_{i}}^{(\tau)}\right), \quad \Sigma_{h_{i}}^{(t)}=\mathrm{S}
$$

where $\mathrm{S}=\left(\sum_{\tau=1}^{t}\left(\hat{\Sigma}_{h_{i}}^{(\tau)}\right)^{-1}\right)^{-1}$, the data from various time stamps are equally weighted in the posterior.

\section{EXPERIMENT}

\subsection{Experimental Setting}

Data. We collect the data from the following public sources. 
- Clinical Trial Records (http://clinicaltrials.gov) includes 360K registered clinical trials. Each record describes a clinical trial in detail. Our experiment include trials that are (1) interventional, (2) tests a single drug, (3) the tested drug is a small molecule drug, and (4) the drug molecule is available in DrugBank. Note that in clinical trial data, diseases are described using unstructured text. We use public API (clinicaltables.nlm.nih.gov) to map them into ICD-10 codes.

- DrugBank (www.drugbank.com) is the database where we acquire drug molecule structures in the format of SMILES (simplified molecular-input line-entry system) strings, a line notation for describing the structure of chemical species using short ASCII strings. - ZINC Molecule Database (zinc.docking.org) has $~ 250 \mathrm{~K}$ druglike molecules [49] and is used to pretrain/train VAE model.

- ICD-10 Coding System (www.icd10data.com) is a medical classification list by the World Health Organization (WHO) [3]. It contains codes for diseases, signs, symptoms, etc. ICD-10 codes are hierarchically represented, where an ancestor of code represents a higher-level category of the current code.

Table 1: Size of selected clinical trials for each time stamp. Note that in 2015-2017 and 2018-2020, we show "\# of drug repurposing samples” + “\# of de novo design samples”.

\begin{tabular}{lccccccc}
\hline Dataset & $00-02$ & $03-05$ & $06-08$ & $09-11$ & $12-14$ & $15-17$ & $18-20$ \\
\hline \# Trials & 1,490 & 3,791 & 5,065 & 5,539 & 5,388 & $5,154+331$ & $4,611+207$ \\
\hline
\end{tabular}

Baselines for Drug Repurposing. We consider the following baselines, LR (Logistic Regression), RF (Random Forest), BPMF (Bayesian Probabilistic Matrix Factorization) [45], DMF (Deep Matrix Factorization) [56], BDMF (Bayesian Deep Matrix Factorization) [53], D-Embed (Dynamic Drug-Disease Embedding) [4], DeepDDI (Deep Drug-Disease Interaction) [40], GraphDDI (Graph DrugDisease Interaction) [13,38] and PRIME-static (without the dynamic model). Table 2 compares the features of these methods.

Table 2: Comparison of various baselines on several aspects.

\begin{tabular}{lcccccccc}
\hline Aspects & LR/RF & BPMF & DMF & BDMF & D-Embed & DeepDDI & GraphDDI & PRIME \\
\hline Neural & - & - & $\checkmark$ & $\checkmark$ & - & $\checkmark$ & $\checkmark$ & $\checkmark$ \\
Uncertainty & - & $\checkmark$ & - & $\checkmark$ & $\checkmark$ & - & - & $\checkmark$ \\
Dynamic & - & - & - & - & $\checkmark$ & - & - & $\checkmark$ \\
\hline
\end{tabular}

\section{Baselines for De Novo Design are listed below:}

- JTVAE (Junction Tree Variational Auto-Encoder) [26]. It leverages junction tree-based VAE to learn the molecular distribution and generate novel molecules from the latent space.

- VJTNN (Variational Junction Tree Neural Network) [25] translation the input molecule into a similar molecule with more desirable molecular properties. Here, given target disease, we use the drugs that can treat target disease as input molecule.

- GCPN (Graph Convolutional Policy Network) [57] leverage graph convolutional network and optimize chemical property of generated molecules through policy gradient.

Implementation Details can be found in Appendix. Code and processed data are available in https://github.com/futianfan/PRIME.
Evaluation Strategy and Metrics. For trial data, we have 7 time stamps (3 year each) from 2000 to 2020 . We use the data 2015-2017 and 2018-2020 to create 2 test sets. We use all the trial samples before the time stamp of the test set to learn the model. The test data are split into two disjoint sets during inference, drug repurposing and de novo design. If the drugs have not been discovered and tested before, the data sample is put into the de novo design. Otherwise, it is categorized into the repurposing set. Table 1 shows the data statistics. Below are the inference strategies for the two tasks.

(1) Drug Repurposing. When we make inferences at time $t$, we attempt to repurpose all the drugs in the existing drug database $\mathcal{M}^{(t-1)}$ at the previous time stamp $t-1$. Two test sets are 2015-2017 and 2018-2020. When predicting at current time window, all the data before the time window are used for training. Each trial has one ground-truth drug. During inference, given the disease code set from each trial, we infer a matching score for each drug in the drug database, as shown in Equation (16). The drug database contains 2,132 drugs by 2015 , and 2,710 drugs by 2018 . Then, we return the ranked drug list based on the matching scores. We consider the following metrics.

- Hit@k accuracy refers to whether the ground-truth drug appears in the first $k$ positions of the ranked drug list.

- Ranking means the ranking of the ground-truth drug in the list. Task Difficulty: The above metrics are challenging to achieve, because we have many repurposing candidates (a few thousands) and the ground-truth drug for a repurposing trial is just one single drug from this set. A simple benchmark for choosing 100 drugs out of this entire repurposing list (for computing hit@100) will lead to $100 / 2,132=4.7 \%$ chance of hitting the correct repurposing drug. As we will see next, PRIME reached $\sim 90 \%$ on this particular metric.

(2) De Novo Drug Design. Following Guacamol Benchmark [10], we design the rediscovery task to mimic de novo design. The target diseases are given as input while the target molecule is unknown for all the methods. We use the trial data before 2018 for training and then test on the trial data in 2018-2020. Our method sample 15K molecules; the first 5k samples are regarded as the burn-in period of SGLD [52]. The burn-in period gives the Markov Chain time to reach its equilibrium distribution, particularly if it has started from a randomly-selected point $[1,52]$. Then we select 500 molecules with the highest QED scores from the remaining $10 \mathrm{~K}$ molecules and compare them with the target molecule. For each target molecule, we fix the number of generated molecules to 500 and report the best results over the 500 generated molecules for each baseline method for a fair comparison. We evaluate using the following metrics.

- Similarity measures Tanimoto similarity between two molecules (generated and target drug molecule) according to [5]. We use RDKit package [30] to evaluate Tanimoto similarity.

- QED (Quantitative Estimate of Drug-likeness) is an integrative score to evaluate molecules' favorability to become a drug. QED score ranges from 0 to 1 and can be evaluated using RDKit package [30]. A higher QED score means more drug-likeness.

- $\log P$ is penalized $\log P$ score from $-\infty$ to $+\infty$. It accounts for ring size and synthetic accessibility [26, 47], higher is better.

- Success Rate is the percentage of successful molecules among all the generated molecules. If one of the generated molecules satisfies (i) the similarity between generated and the target molecule is 
higher than 0.30 and (ii) the QED score is higher than 0.70 , it is considered successfully generated, following [25, 59].

\subsection{Results on Drug Repurposing}

From results in Table 3, PRIME consistently outperforms all baselines in all metrics across two test sets. Compared with the best baseline GraphDDI, PRIME obtains $27.4 \%$ and $24.4 \%$ relative reduction in the average ranking of the ground-truth drug, $6.9 \%$ and 5.3\% absolute improvement on hit@100 accuracy on 2015-2017 and 2018-2020 test sets, respectively. Also, we report the p-values to measure the statistical difference between PRIME and the best baseline GraphDDI, the p-values are 0.018 and 0.013 , below 0.05 threshold, which showcases the statistical significance of our method over the best baseline. Both methods use the same neural architecture; the difference is PRIME uses a dynamic probabilistic model to quantify uncertainty and capture evolving molecule-disease relations. PRIME also significantly reduce the search space. For example, its hit@100 accuracy on the $2015-2017$ test set is $93.9 \%$ (i.e., $93.9 \%$ of repurposing samples in the test set), which means our prediction set almost always contains the ground-truth molecule.

Table 3: Drug Repurposing results measured by hit@ $k$ accuracy (higher the better) and ranking (lower the better). For ranking, the p-values between PRIME and graphDDI (the best baseline) are 0.018 and 0.013 on the two corresponding time windows, confirming its statistical significance.

\begin{tabular}{lcccccc}
\hline \multicolumn{3}{c}{ 2015-2017 (2,132 drugs) } & \multicolumn{3}{c}{ 2018-2020 (2,710 drugs) } \\
Method & hit@50 & hit@100 & ranking & hit@50 & hit@100 & ranking \\
\hline LR & $40.4 \%$ & $62.4 \%$ & $160.4 \pm 27.3$ & $38.5 \%$ & $59.2 \%$ & $181.0 \pm 24.4$ \\
RF & $41.1 \%$ & $63.7 \%$ & $155.6 \pm 20.6$ & $39.1 \%$ & $60.0 \%$ & $173.5 \pm 21.6$ \\
BPMF & $42.3 \%$ & $66.1 \%$ & $140.2 \pm 21.3$ & $41.3 \%$ & $64.6 \%$ & $167.2 \pm 16.7$ \\
DMF & $48.5 \%$ & $85.6 \%$ & $93.3 \pm 19.7$ & $49.8 \%$ & $79.3 \%$ & $118.4 \pm 16.5$ \\
BDMF & $49.6 \%$ & $87.5 \%$ & $82.1 \pm 20.5$ & $52.6 \%$ & $84.2 \%$ & $93.2 \pm 12.8$ \\
D-Embed & $43.3 \%$ & $72.6 \%$ & $121.3 \pm 19.0$ & $45.6 \%$ & $71.1 \%$ & $129.0 \pm 13.2$ \\
DeepDDI & $46.6 \%$ & $80.4 \%$ & $89.6 \pm 16.4$ & $51.9 \%$ & $82.2 \%$ & $110.2 \pm 14.5$ \\
GraphDDI & $49.7 \%$ & $87.0 \%$ & $81.2 \pm 14.3$ & $52.9 \%$ & $84.3 \%$ & $90.6 \pm 13.8$ \\
PRIME-static & $50.2 \%$ & $88.2 \%$ & $78.4 \pm 12.6$ & $53.2 \%$ & $84.9 \%$ & $82.8 \pm 13.5$ \\
PRIME & $\mathbf{5 2 . 9 \%}$ & $\mathbf{9 3 . 9 \%}$ & $\mathbf{5 9 . 0 \pm 1 1 . 8}$ & $\mathbf{5 6 . 2 \%}$ & $\mathbf{8 9 . 6 \%}$ & $\mathbf{6 8 . 2 \pm 1 2 . 4}$ \\
\hline
\end{tabular}

We also use case studies in Fig. 2 to show the evolving moleculedisease relations from 2000 to 2017 . We select several common drugs to treat lung cancer and Alzheimer's disease (AD) and observe the changes in their molecule-disease matching score (Eq. 15). From Fig. 2(a), we observe Erlotinib becomes more popular in treating lung cancer. In contrast, Pemetrexed gradually phased out due to relatively worse effectiveness, consistent with the observational study [21]. Fig. 2(b) demonstrates that Donepezil gradually exhibits its superiority in treating $\mathrm{AD}$ while Galantamine gradually becomes less popular, consistent with [7].

\subsection{Results on De Novo Drug Design}

From the results in Table 4, PRIME achieves the best performance in similarity score, LogP score and success rate. All methods achieve desirable QED scores, validating their effectiveness in generalpurpose de novo drug design. VJTNN achieves the highest QED score since it is designed to optimize QED score. Unlike GCPN and VJTNN that explicitly optimize QED and LogP, PRIME still get competitive performance in QED and LogP because it learns from high-quality drug molecules in clinical trial. Compared with other methods, PRIME is able to find the similar molecular structure

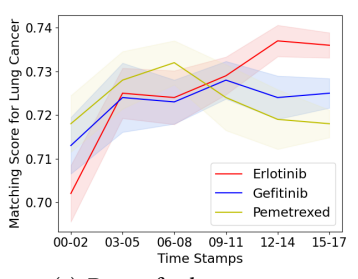

(a) Drugs for lung cancer

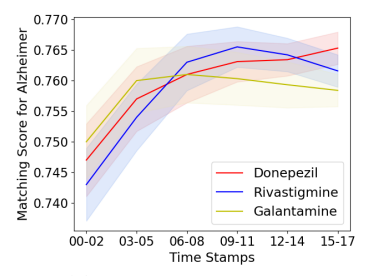

(b) Drugs for Alzheimer
Figure 2: Case study to illustrate the evolution of some molecule-disease relations from 2000 to 2017.)

with the target drug because the disease-oriented posterior (Eq. 17) narrow down the chemical space. To investigate why PRIME outperforms the general-purpose de novo design methods, we conduct the following case studies.

Table 4: Results for Disease-oriented de novo design.

\begin{tabular}{lcccc}
\hline Method & Similarity & QED & LogP & Success Rate \\
\hline JTVAE [26] & 0.160 & 0.732 & 0.27 & $2.3 \%$ \\
GCPN [57] & 0.170 & 0.747 & 0.96 & $6.8 \%$ \\
VJTNN [25] & 0.214 & 0.815 & 0.75 & $16.4 \%$ \\
PRIME-static & 0.318 & 0.781 & 1.02 & $21.3 \%$ \\
PRIME & 0.356 & 0.783 & 0.97 & $24.2 \%$ \\
\hline
\end{tabular}

In Fig. 3, we show some sample outputs. Both Evenamide and Pyrazinamide are exactly rediscovered. We can also generate many molecule candidates that are very similar to the rediscovered drug and with desirable drug-like properties measured by QED score. The is due to PRIME draws samples from the posterior defined in Eq. (17), which captures the uncertainty in chemical space. This is very useful in real-world de novo drug design.

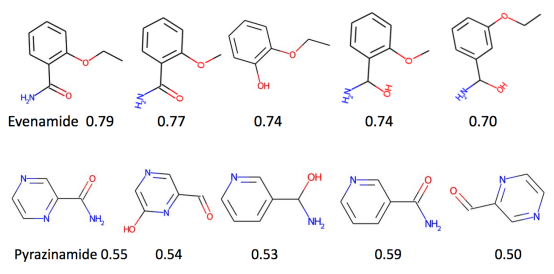

Figure 3: Evenamide and Pyrazinamide are rediscovered. PRIME also generates similar ones with desirable QED score.

Then in Fig. 4, we visualize the chemical space via reducing VAE's latent space into 2D points. Concretely, we map all molecules into latent variables using JTVAE's encoder and leverage PCA for dimensional reduction. Here gray points are generated by JTVAE, the general de novo design method. These generated molecules are disease agnostic and mostly far from the target. Blue points are the posterior samples of PRIME via MCMC sampler (SGLD [52] here). Since PRIME draws samples from the posterior defined in Eq. (17), which constrains the chemical space, we can generate molecules that are close to the target molecule. The light blue points are samples in MCMC burn-in period. Starting from a randomlyselected point, the MCMC sampler needs a burn-in period to reach 
its equilibrium distribution. The yellow points are generated by VJTNN, the best baseline. The red point corresponds to Evenamide, the target molecule. We find that the target molecule Evenamide lies in the blue points cloud, showing that PRIME effectively narrows down the search space, and could save time and resources.

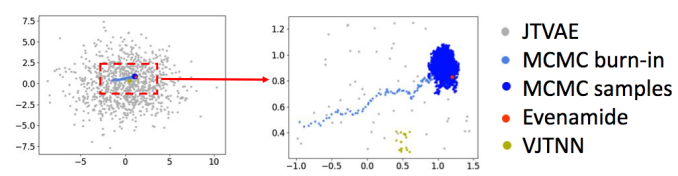

Figure 4: Chemical space when rediscovering Evenamide. MCMC samples from PRIME are very close to the target drug (red), while the samples from other baselines (JTVAE, VJTNN) are scattered farther away from the target.

\subsection{External Validation Using Real-World Observational Data}

Following previous studies [34, 54, 55], we conduct an association study of PRIME on disease-oriented drug repurposing using realworld medical claims data. The basic idea is to produce top-ranked disease-drug pairs, and compare patients who take the drug (case group) vs. ones who do not (control group) on the disease related metrics. If the case group performs better, it suggests the drug might have efficacy in treating the target disease. We extract patient data from a large US claims database from Jan. 2017 to Dec. 2020,which contains encrypted patient's ID, zip code, date of claims, diagnosis code (i.e., ICD-9/10 codes), prescription drug. It includes 329,937,786 patients and 115,806 ICD-9/10 codes.

Study design. We use a hybrid case-control design to construct the patient cohort and propensity matching to bin the patients into comparable risk groups. Given the repurposed drug $m$ and target disease code $d$, a patient is assigned to the case group if the patient (a) consistently had the drug $m$ since 2018; and (b) had drug $m$ in the claim for at least 6 times; and (c) had follow-up diagnosis records $d$ after the first time that $m$ was recorded. The control group are matched using nested case-control study disease if the patient did not take the repurposed drug during 2017 and 2020. A control patient is matched with a case patient on zip code, gender, birthyear, with claims data in the same time period as the case. The index date for a case patient is the first date of using drug $m$, while the index date for a control is the index date of the matching case.

We use propensity scores to assign cases and controls into comparable risk groups, following [34, 54]. To compute propensity scores, we train a logistic regression classifier for the outcome label for all cases and controls. The outcome label is whether a case or control will have the target disease throughout of the diagnosis claims records. The features of logistic regression are binary variables of all other diagnoses (excluding the target diseases). We define the propensity score as the class probability generated by the logistic regression classifier. We further divide case and control patients into three risk bins based on their propensity scores, $[0,0.1),[0.1,0.3),[0.3,1]$.

Evaluation metric. We used the Average Treatment Effect (ATE) as the evaluation metric at the population level. ATE for each risk bin is defined as ATE $=\mathbb{E}\left(P_{\text {case }}\right)-\mathbb{E}\left(P_{\text {control }}\right)$, where $\mathbb{E}\left(P_{\text {case }}\right)$ and $\mathbb{E}\left(P_{\text {control }}\right)$ represents the expected case and control outcomes, respectively. Specifically, given a repurposed drug and a set of disease ICD codes, patients in the case and control groups are labeled based on whether the patient will have the target disease starting 30 days after the index date, where " 1 " means have and " 0 " means not. For ATE, the smaller (i.e., negative ATE), the better.

Results. We compute the p-value using the McNemar's Test [14, 34] to measure the statistical significance of ATE between case and control groups. We perform the one-sided McNemar's Test [14] with null hypothesis being the marginal probability of the case group (i.e., case ATE) greater than that of the control group. We tested 278 drug-disease pairs that both case and control group have at least 100 patients, among which 199 pairs have negative ATE, indicating that the patients taking the repurposed drug had a lower risk of having the disease than those who did not. Also, 21 pairs have significance results ( $\mathrm{p}$-value $<0.05$ ). We list some sample results in Table 5, supported by literatures.

Table 5: External Validation of Drug Repurposing.

\begin{tabular}{cccccc}
\hline Drug & Disease & ATE & p-value & \# case:\# control & literature \\
\hline Dutasteride & prostate cancer & -0.07 & $9.6 \mathrm{e}-4$ & $1049: 1049$ & {$[2]$} \\
Ramipril & hypertrophic cardiomyopathy & -0.04 & 0.0016 & $1696: 1696$ & {$[36]$} \\
Clopidogrel & obstructive hydrocephalus & -0.04 & $1.2 \mathrm{e}-5$ & $3794: 3794$ & {$[50]$} \\
\hline
\end{tabular}

\subsection{Ablation and Model Variants Study}

We also conduct an ablation study on drug repurposing.

- PRIME-MLE: an optimization-based variant without quantifying uncertainty. Concretely, unlike PRIME that estimates $\mu_{h}$ and $\Sigma_{h}$ and then draws $\mathbf{h} \sim \mathcal{N}\left(\mu_{h}, \Sigma_{h}\right)$, in PRIME-MLE $\mathbf{h}$ is the MPN's output. Maximum likelihood estimation (MLE) is used for optimization.

- PRIME-static: the static version of PRIME. During training, all samples with different time stamps are used in the posterior.

- PRIME-weight: we train two models on current data $\mathcal{X}^{(t)}$ and previous data $X^{\leq}(t-1)$ and ensemble them following in Equation (19). $\sigma_{1}^{2}, \sigma_{2}^{2}$ weigh the importance of previous data $X^{\leq(t-1)}$. From $\sigma_{1}^{2}, \sigma_{2}^{2} \in$ $\{0.1,1,10\}$, we set $\sigma_{1}^{2}=\sigma_{2}^{2}=1$, which achieve best performance. - PRIME-ME (mean embedding): in Eq. (5), the likelihood function is the geometric mean of the likelihood of various drug-disease pairs, i.e., $\prod_{d \in D_{k}} \sigma\left(\mathbf{h}_{m_{x_{i}}}^{\top} \mathbf{v}_{d}\right)^{\frac{1}{\left|D_{k}\right|}}$. We compare it with another variant, $\sigma\left(\mathbf{h}_{m_{x_{i}}}^{\top}\left(\frac{1}{\left|D_{k}\right|} \sum_{d \in D_{k}} \mathbf{v}_{d}\right)\right)$.

- PRIME-MS (matching score): when evaluating matching score in Eq. (16), PRIME use $\mu_{s}(m)-\gamma \sigma_{s}(m)$ to replace the mean $\mu_{s}(m)$. To explore its empirical impact, we include the variant PRIME-MS that uses $\mu_{s}(m)$ as matching score.

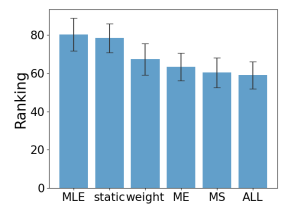

(a) $2015-2017$

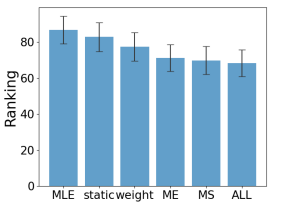

(b) $2018-2020$
Figure 5: Ablation Study.

We show the results in Fig. 5. "ALL" keeps all the components, i.e., PRIME itself. We observe changing each component would cause 
a decrease in accuracy more or less on both test sets. Among all these variants, PRIME-MLE achieves the lowest accuracy, showing the necessity of using uncertainty estimation. Also, PRIME-static leads to a significant loss in accuracy, which implies the importance of modeling evolving molecule-disease relations. PRIME-weight outperforms PRIME-static, which empirically validates the fact that putting more weight on current data does enhance the performance, as shown in Theorem 1.

\section{CONCLUSION}

In this paper, we proposed a probabilistic and dynamic deep learning (PRIME) method to tackle drug repurposing and de novo design tasks using high quality clinical trial data. PRIME models evolving drugdiseases relations and capturing model uncertainty. We evaluated PRIME on drug repurposing and de novo design tasks using realworld clinical trial data and demonstrated the potential efficiency and effectiveness of PRIME in drug discovery.

\section{CONTRIBUTION STATEMENT}

TF and JS proposed the method, and CX, CQ and LMG contributed to the experiments.

\section{ACKNOWLEDGEMENTS}

This work was supported by NSF award SCH-2014438, PPoSS 2028839 , IIS-1838042, NIH award R01 1R01NS107291-01 and OSF Healthcare.

\section{REFERENCES}

[1] Sungjin Ahn, Anoop Korattikara, and Max Welling. 2012. Bayesian posterior sampling via stochastic gradient fisher scoring. In ICML.

[2] Gerald Andriole et al. 2010. Effect of dutasteride on the risk of prostate cancer. N Engl f Med (2010)

[3] Stefan D Anker et al. 2016. Welcome to the ICD-10 code for sarcopenia. Fournal of cachexia, sarcopenia and muscle (2016).

[4] Robert Bamler and Stephan Mandt. 2017. Dynamic Word Embeddings. In ICML.

[5] Igor Baskin et al. 2009. Fragment descriptors in SAR / QSAR / QSPR studies, molecular similarity analysis and in virtual screening. ChemInform (2009).

[6] Charles Blundell et al. 2015. Weight uncertainty in neural network. In ICML.

[7] Mary Bond et al. 2013. The effectiveness and cost-effectiveness of donepezil, galantamine, rivastigmine and memantine for the treatment of Alzheimer's disease. Health technology assessment (2013).

[8] George EP Box and George C Tiao. 2011. Bayesian inference in statistical analysis.

[9] James R Broach, Jeremy Thorner, et al. 1996. High-throughput screening for drug discovery. Nature 384, 6604 (1996), 14-16.

[10] Nathan Brown et al. 2019. GuacaMol: benchmarking models for de novo molecular design. Journal of chemical information and modeling (2019).

[11] Nicola De Cao and Thomas Kipf. 2018. MolGAN: An implicit generative model for small molecular graphs. arXiv:1805.11973

[12] Huiyuan Chen and Jing Li. 2017. A flexible and robust multi-source learning algorithm for drug repositioning. In $A C M-B C B$. 510-515.

[13] Huiyuan Chen and Jing Li. 2020. Learning Data-Driven Drug-Target-Disease Interaction via Neural Tensor Network. IfCAI (2020).

[14] Michael R Chernick et al. 2011. Bootstrap methods.

[15] Edward Choi et al. 2017. GRAM: graph-based attention model for healthcare representation learning. In $K D D$.

[16] Hanjun Dai et al. 2016. Discriminative embeddings of latent variable models for structured data. In ICML.

[17] Tianfan Fu et al. 2020. CORE: Automatic Molecule Optimization using Copy and Refine Strategy. AAAI (2020).

[18] Tianfan Fu et al. 2021. MIMOSA: Multi-constraint Molecule Sampling for Molecule Optimization. AAAI (2021)

[19] Peter D Gluckman and Mark A Hanson. 2004. Living with the past: evolution, development, and patterns of disease. Science (2004).

[20] Rafael Gómez-Bombarelli et al. 2018. Automatic chemical design using a datadriven continuous representation of molecules. ACS central science (2018).

[21] Xiaohua Gu et al. 2019. Cost-effectiveness of afatinib, gefitinib, erlotinib and pemetrexed-based chemotherapy as first-line treatments for advanced non-small cell lung cancer. Lung Cancer (2019).
[22] Markus Hartenfeller et al. 2010. De novo drug design. Chemoinformatics (2010).

[23] Kexin Huang et al. 2020. DeepPurpose: A Deep Learning Library for Drug-Target Interaction Prediction. Bioinformatics (2020).

[24] Kexin Huang et al. 2021. Therapeutics Data Commons: Machine Learning Datasets and Tasks for Therapeutics. arXiv:2102.09548 (2021).

[25] Wengong Jin et al. 2019. Learning Multimodal Graph-to-Graph Translation for Molecular Optimization. ICLR (2019).

[26] Wengong Jin, Regina Barzilay, and Tommi Jaakkola. 2018. Junction tree variational autoencoder for molecular graph generation. ICML (2018).

[27] Alistair EW Johnson et al. 2016. MIMIC-III, a freely accessible critical care database. Scientific data (2016).

[28] Thomas N Kipf and Max Welling. 2017. Semi-supervised classification with graph convolutional networks. ICLR (2017).

[29] Matt J Kusner et al. 2017. Grammar variational autoencoder. In ICML.

[30] Greg Landrum et al. 2006. RDKit: Open-source cheminformatics.

[31] Jiao Li et al. 2016. A survey of current trends in computational drug repositioning. Briefings in bioinformatics 17, 1 (2016), 2-12.

[32] Jaechang Lim et al. 2019. Predicting drug-target interaction using a novel graph neural network with 3D structure-embedded graph representation. Fournal of chemical information and modeling 59, 9 (2019), 3981-3988.

[33] Qi Liu, Miltiadis Allamanis, Marc Brockschmidt, and Alexander Gaunt. 2018. Constrained graph variational autoencoders for molecule design. In NeurIPS

[34] Ruoqi Liu et al. 2021. A deep learning framework for drug repurposing via emulating clinical trials on real-world patient data. Machine Intelligence (2021).

[35] Ben London, Bert Huang, and Lise Getoor. 2015. The benefits of learning with strongly convex approximate inference. In ICML.

[36] Kristin MacDonald et al. 2006. The effect of ramipril on left ventricular mass, diastolic function, and plasma neurohormones in Maine Coon cats with hypertrophic cardiomyopathy. Fournal of veterinary internal medicine (2006).

[37] Tomas Mikolov et al. 2013. Distributed representations of words and phrases and their compositionality. NIPS (2013).

[38] Thin Nguyen et al. 2019. GraphDTA: prediction of drug-target binding affinity using graph convolutional networks. Bioinformatics (2019).

[39] AkshatKumar Nigam et al. 2020. Augmenting Genetic Algorithms with Deep Neural Networks for Exploring the Chemical Space. In ICLR.

[40] Hakime Ozturk et al. 2018. DeepDTA: deep drug-target binding affinity prediction. Bioinformatics (2018).

[41] Pavel G Polishchuk et al. 2013. Estimation of the size of drug-like chemical space based on GDB-17 data. Fournal of computer-aided molecular design (2013).

[42] Daniil Polykovskiy et al. 2020. Molecular sets (MOSES): a benchmarking platform for molecular generation models. Frontiers in pharmacology (2020).

[43] Yuan Quan et al. 2018. Evolutionary and genetic features of drug targets. Medicinal research reviews (2018).

[44] Anna Rutkowska et al. 2016. A modular probe strategy for drug localization, target identification and target occupancy measurement on single cell level. ACS Chemical Biology (2016).

[45] Ruslan Salakhutdinov and Andriy Mnih. 2008. Bayesian probabilistic matrix factorization using Markov chain Monte Carlo. In ICML.

[46] Robert P Sheridan. 2013. Time-split cross-validation as a method for estimating the goodness of prospective prediction. fournal of chemical information and modeling 53, 4 (2013), 783-790.

[47] Chence Shi et al. 2019. GraphAF: a Flow-based Autoregressive Model for Molecular Graph Generation. In ICLR.

[48] Martin Simonovsky and Nikos Komodakis. 2018. GraphVAE: Towards generation of small graphs using variational autoencoders. In ICANN.

[49] Teague Sterling and John J Irwin. 2015. ZINC 15-ligand discovery for everyone. fournal of chemical information and modeling (2015).

[50] Y Tamura et al. 2014. Aspirin/clopidogrel. Reactions (2014).

[51] Chong Wang et al. 2008. Continuous time dynamic topic models. In UAI.

[52] Max Welling and Yee W Teh. 2011. Bayesian learning via stochastic gradient Langevin dynamics. In ICML. 681-688.

[53] Teng Xiao et al. 2020. Bayesian deep collaborative matrix factorization. In AAAI.

[54] Hua Xu et al. 2015. Validating drug repurposing signals using electronic health records: a case study of metformin associated with reduced cancer mortality. FAMIA (2015).

[55] Hua Xu et al. 2020. Electronic health records for drug repurposing: Current status, challenges, and future directions. CPT (2020).

[56] Hong-Jian Xue et al. 2017. Deep Matrix Factorization Models for Recommender Systems. In IFCAI.

[57] Jiaxuan You et al. 2018. Graph Convolutional Policy Network for Goal-directed Molecular Graph Generation. In NIPS.

[58] Yaxia Yuan et al. 2020. Ligbuilder v3: a multi-target de novo drug design approach. Frontiers in chemistry (2020).

[59] Chengxi Zang and Fei Wang. 2020. MoFlow: an invertible flow model for generating molecular graphs. In $K D D$.

[60] Cheng Zhang et al. 2018. Advances in variational inference. TPAMI (2018).

[61] Alex Zhavoronkov. 2018. Artificial intelligence for drug discovery, biomarker development, and generation of novel chemistry. 


\section{A DETAILS ABOUT THEORETICAL RESULTS}

In this section, we show brief proofs for theoretical analysis.

Lemma 4. Let $\mathcal{N}_{x}(\mathbf{m}, \Sigma)$ be the density of $x$ for Gaussian distribution with mean $\mathbf{m}$ and variance $\Sigma$, then the product of two Gaussian density is also a Gaussian density, we have

$$
\begin{aligned}
& \mathcal{N}_{x}\left(\mathbf{m}_{3}, \Sigma_{3}\right) \propto \mathcal{N}_{x}\left(\mathbf{m}_{1}, \Sigma_{1}\right) \cdot \mathcal{N}_{x}\left(\mathbf{m}_{2}, \Sigma_{2}\right) \\
& \mathbf{m}_{3}=\left(\Sigma_{1}^{-1}+\Sigma_{2}^{-1}\right)^{-1}\left(\Sigma_{1}^{-1} \mathbf{m}_{1}+\Sigma_{2}^{-1} \mathbf{m}_{2}\right), \quad \Sigma_{3}=\left(\Sigma_{1}^{-1}+\Sigma_{2}^{-1}\right)^{-1} .
\end{aligned}
$$

The normalizing constant is ignored for simplicity.

Proof. We ignore the constant that does not involve $x$ and have

$$
\begin{aligned}
\exp ( & \left.-\frac{1}{2}\left[\left(x-\mathbf{m}_{1}\right)^{\top} \Sigma_{1}^{-1}\left(x-\mathbf{m}_{1}\right)+\left(x-\mathbf{m}_{2}\right)^{\top} \Sigma_{2}^{-1}\left(x-\mathbf{m}_{2}\right)\right]\right) \\
\propto & \exp \left(-\frac{1}{2}\left[x^{\top}\left(\Sigma_{1}^{-1}+\Sigma_{1}^{-1}\right) x-2 x^{\top}\left(\Sigma_{1}^{-1} \mathbf{m}_{1}+\Sigma_{2}^{-1} \mathbf{m}_{2}\right)\right]\right) \\
\propto & \exp \left(-\frac{1}{2} x^{\top}\left(\left(\Sigma_{1}^{-1}+\Sigma_{1}^{-1}\right)^{-1}\right)^{-1} x\right. \\
& \left.\quad-2 x^{\top}\left(\Sigma_{1}^{-1}+\Sigma_{2}^{-1}\right)\left(\Sigma_{1}^{-1}+\Sigma_{2}^{-1}\right)^{-1}\left(\Sigma_{1}^{-1} \mathbf{m}_{1}+\Sigma_{2}^{-1} \mathbf{m}_{2}\right)\right) \\
\propto & \exp \left(-\frac{1}{2}\left(x-\left(\Sigma_{1}^{-1}+\Sigma_{2}^{-1}\right)^{-1}\left(\Sigma_{1}^{-1} \mathbf{m}_{1}+\Sigma_{2}^{-1} \mathbf{m}_{2}\right)\right)^{\top}\right. \\
& \left.\quad\left(\left(\Sigma_{1}^{-1}+\Sigma_{2}^{-1}\right)^{-1}\right)^{-1}\left(x-\left(\Sigma_{1}^{-1}+\Sigma_{2}^{-1}\right)^{-1}\left(\Sigma_{1}^{-1} \mathbf{m}_{1}+\Sigma_{2}^{-1} \mathbf{m}_{2}\right)\right)\right) \\
= & \exp \left(-\frac{1}{2}\left(x-\mathbf{m}_{3}\right)^{\top} \Sigma_{3}^{-1}\left(x-\mathbf{m}_{3}\right)\right)
\end{aligned}
$$

Proved.

Lemma 5. If a probability distribution $P_{2}$ satisfies

$$
\mathcal{N}_{x}\left(\mathbf{m}_{3}, \Sigma_{3}\right) \propto \mathcal{N}_{x}\left(\mathbf{m}_{1}, \Sigma_{1}\right) \cdot P_{2} \text {, and } \Sigma_{1}>\Sigma_{3}>0,
$$

then we have $P_{2} \sim \mathcal{N}\left(\mathbf{m}_{2}, \Sigma_{2}\right)$, where

$$
\mathbf{m}_{2}=\Sigma_{2}\left[\left(\Sigma_{1}^{-1}+\Sigma_{2}^{-1}\right) \mathbf{m}_{3}-\Sigma_{1}^{-1} \mathbf{m}_{1}\right], \quad \Sigma_{2}=\left(\Sigma_{3}^{-1}-\Sigma_{1}^{-1}\right)^{-1}>0 .
$$

Square matrix $Z>0$ means $Z$ is positive definite.

Proof. Suppose $P_{2}$ is the Gaussian distribution as described in Equation (5), then via Lemma 4, we find $\mathcal{N}\left(\mathbf{m}_{3}, \Sigma_{3}\right)$ is exactly the density of $\mathcal{N}_{x}\left(\mathbf{m}_{1}, \Sigma_{1}\right) \cdot P_{2}$. Since $\Sigma_{1}, \Sigma_{2}, \Sigma_{3}$ are all non-singular, $P_{2}$ is unique.

Lemma 3 is based on Lemma 5. Specifically, it is easy to satisfying the condition that $\Sigma_{1}>\Sigma_{3}>0$ via choosing the appropriate $\sigma_{1}, \sigma_{2}$ in Equation (9). Theorem 1 is proved by directly using Lemma 4. For Corollary 1 , when $\sigma_{1}^{2} \rightarrow \infty$, we have

$$
\lim _{\sigma_{1}^{2} \rightarrow+\infty} \frac{1}{\sigma_{1}^{2}} I \rightarrow 0, \quad \lim _{\sigma_{1}^{2} \rightarrow+\infty}\left(\Sigma_{h_{i}}^{(t-1)}\right)^{-1}+\frac{1}{\sigma_{1}^{2}} I=\left(\Sigma_{h_{i}}^{(t-1)}\right)^{-1} .
$$

Then we iteratively use Lemma 4 and get

$$
\mu_{h_{i}}^{(t)}=\left(\sum_{t=1}^{T}\left(\hat{\Sigma}_{h_{i}}^{(t)}\right)^{-1}\right)^{-1}\left(\sum_{t=1}^{T}\left(\hat{\Sigma}_{h_{i}}^{(t)}\right)^{-1} \hat{\mu}_{h_{i}}^{(t)}\right) ; \quad \Sigma_{h_{i}}^{(t)}=\left(\sum_{t=1}^{T}\left(\hat{\Sigma}_{h_{i}}^{(t)}\right)^{-1}\right)^{-1}
$$

For simplicity, we abuse the notation a bit and use $q(\Theta ; \Psi)$ to replace $q\left(\Theta ; f\left(\mathcal{X}^{(t)} ; \Psi\right)\right)$ For Lemma 1, we have

$$
\begin{aligned}
& \mathcal{L}(\Psi)=\mathrm{KL}\left(q(\Theta ; \Psi) \| p\left(\Theta \mid \mathcal{X}^{(t)}\right)\right) \\
= & \int q(\Theta ; \Psi)\left[\log q(\Theta ; \Psi)-\log p\left(\Theta^{(t)}\right)-\log p\left(\mathcal{X}^{(t)} \mid \Theta^{(t)}\right)\right] d \theta \\
= & \int q(\Theta ; \Psi)\left[\log \frac{q(\Theta ; \Psi)}{p(\Theta(t)}-\log p\left(\mathcal{X}^{(t)} \mid \Theta^{(t)}\right)\right] d \theta \\
= & \underbrace{\operatorname{KL}\left(q\left(\Theta ; f\left(\mathcal{X}^{(t)} ; \Psi\right)\right) \| p\left(\Theta^{(t)}\right)\right)}_{\mathcal{L}_{1}(\Psi)}-\underbrace{\mathbb{E}_{q\left(\Theta ; f\left(\mathcal{X}^{(t)} ; \Psi\right)\right)}\left[\log p\left(\mathcal{X}^{(t)} \mid \Theta\right)\right]}_{\mathcal{L}_{2}(\Psi)}
\end{aligned}
$$

In derivation, we ignore the term that are not related to $\Psi$.

For Lemma 2, we have

$$
\begin{aligned}
& \mathcal{L}_{1}(\Psi)=\mathrm{KL}\left(q(\Theta ; \Psi) \| p\left(\Theta^{(t)}\right)\right)=\int q(\Theta ; \Psi)\left[\log \frac{q(\Theta ; \Psi)}{p\left(\Theta^{(t)}\right)}\right] d \theta \\
= & \int q(\Theta ; \Psi)\left[\log q(\Theta ; \Psi)-\log \mathcal{N}\left(0, \sigma_{1}^{2} I\right)-\log q\left(\Theta ; \Psi^{(t-1)}\right)\right] d \theta \\
= & \int q(\Theta ; \Psi)\left[\log q\left(\Theta ; \Psi^{(t-1)}\right)-\log q\left(\Theta ; \Psi^{(t-1)}\right)\right] d \theta \\
\leq & L \int p(\Theta ; \Psi)\left\|\Psi-\Psi^{(t-1)}\right\| d \theta=L\left\|\Psi-\Psi^{(t-1)}\right\|,
\end{aligned}
$$

where the inequality follows from Lipschitz condition in Assumption 1.

\section{B REPRODUCIBILITY}

\section{B.1 Reproducibility of PRIME}

We describe the implementation details of PRIME as follows.

- (i) molecule VAE. Concretely, PRIME use neural architecture of JTVAE (Junction Tree Variational Auto-Encoder) [25, 26]. Most of the setup follows JTVAE $[25,26]$. To construct cycle-free structure and simplify the molecule generation procedure, JTVAE first convert molecular graph into junction tree, where the ring in original molecular graph becomes a node in junction tree. The raw atom features is a 39 dimension vector, including its atom/ring type, degree, its formal charge and its chiral configuration. The raw bond feature is a concatenation of its bond type (4 dimensional onehot vector, indicating whether it is single, double, triple, aromatic bond), whether the bond is in a ring, and its cis-trans configuration. Like conventional VAE, JTVAE has two parts: encoder and decoder. Both encoder and decoder have junction tree and molecular graph phase. Both junction tree encoder and molecular graph encoder leverage graph message passing network (MPN) $[16,26]$ to learn the representation, where the depth are 6 and 3, respectively. RELU is used as activation function. Mean function is used as the readout function for MPN. JTVAE is pretrained on ZINC database (https://zinc.docking.org/), which is publicly available and contains about $250 \mathrm{~K}$ drug-like molecules [49]. The ratio of training to validation data samples is 9. During pretraining, the learning epoch is set to 10. Adam optimizer is used with initial learning rate $1 e^{-3}$. Every epoch, the learning rate is annealed by 0.8 . The convergence criteria is the reconstruction rate (exactly recovered) on validation set. Since the data size is large, every 0.1 epoch, we check the convergence criteria to avoid overfitting. 
- (ii) disease encoder We use ICD-10 code [3] to represent disease code. It usually expresses the hierarchy of various medical concepts in the form of a parent-child relationship, e.g., "C34" (malignant neoplasm of bronchus and lung) and "C 34.9" (malignant neoplasm of unspecified part of bronchus or lung) are the ancestors of "C34.91" (malignant neoplasm of right bronchus or lung). Disease code representation is designed to utilize the knowledge hidden in the hierarchical information in medical ontologies [15], as shown in Equation (3). In Equation (3), to compute attention weight, we use a feed-forward network with a two-layer neural network with hidden dimension 20 and 1 . We use co-occurrence information to learn the basic embeddings of medical codes and their ancestors to initalize the basic embedding of medical codes $\mathbf{e}$ Co-occurrence has proven to be effective when learning representations of words or medical concepts [15]. Worth to mention that different from word embedding that specify a sliding window to define co-occurrence, for medical code, the co-occurrences mean the different medical codes appear in a visit. We pretrain disease embedding using MIMIC-III dataset [27] following [15]. MIMIC-III dataset consists of electronic records of $7.5 \mathrm{~K}$ intensive care unit (ICU) patients over 11 years. We utilize all the patients with at least 2 visits.

- (iii) variational inference After pretraining both molecule VAE and disease embedding, we are able to build PRIME and learn the whole model. Based on the trial samples, we can estimate the gradient of molecule VAE and disease embedding as shown in Equation (14). We use Adam optimizer to optimize the variational objective and the initial learning rate is set to 0.001 . Maximal epoch is set to 10 . The regularization hyperparameter $\lambda$ defined in Equation (13) is set to 0.5 . These hyperparameter are tuned on learning 2012-2014 time window as validation set, (2000-2011 as training).

- (iv) Evaluation on drug repurposing For each repurposed drug molecule $m$, we have the approximate posterior $q\left(\mathbf{h}_{m}^{(\tau-1)}\right), q\left(\mathbf{v}_{i}^{(\tau-1)}\right)$, we are interested in evaluating the expectation of their matching score under the posterior distributions in Equation (15). We use the Monte Carlo samples to estimate $\widehat{\mathcal{S}^{(t)}}\left(m, D_{x}\right)$. Concretely, we sample for each tested drug, we draw 100 Monte Carlo samples to evaluate the matching score.

- (v) Evaluation on de novo drug Design. We leverage Stochastic Gradient Langevin Dynamics (SGLD) [52]. In order to satisfy Robbins-Monro conditions [52], we set stepsize of SGLD as $\epsilon_{t}=$ $\frac{1}{10 *(10000+t)^{0.51}}$, which decays polynomially, following rule of thumb [52] and guarantee the theoretical convergence to the true posterior. Hardware and Software Setup. All the experiments are conducted on an Intel Xeon E5-2690 machine with 256G RAM and 8 NVIDIA Pascal Titan X GPUs. Our method is implemented by Python 3.7 and Pytorch 1.7.1. RDKit version has to be later than 2019.11. Both the code and processed data are attached.

\section{B.2 Details on baseline methods}

First, we describe the technical details about baseline methods for drug repurposing. We use Adam optimizer with initial learning rate 0.001 for all neural network based models. The dimension of drug and disease representations are both 100 for all methods. Following $[4,53,56]$, the ratio of negative-to-positive sample is set to 1 for all methods. For NN based approaches, the batch size is set to 64, RELU is used as the default activation function in hidden layer. In output layer for binary prediction, sigmoid is used as default activation function. Maximal epoch number is set to 10 for all approaches.

- LR (Logistic Regression) and RF (Random Forest): It concatenate drug molecular feature (1024-bit ECFC6 Morgan fingerprint [5]) and multi-hot vector that encodes ICD-10 codes. We leverage Python scikit-learn package for LR and RF.

- BPMF (Bayesian Probabilistic Matrix Factorization) [45]. It represent historical clinical trial into Drug-Disease matrix as recommendation system and leverage Bayesian Probabilistic Matrix Factorization (BPMF) to infer the factor variables for both drug and disease. The likelihood function is also Equation (5). Each factor variable is imposed a Gaussian prior with zero-mean and unit variance. The dimension of factor variables is set to 100. MAP (maximum a posteriori) is leveraged to update drug factor variable and disease factor variable alternatively like Gibbs sampling.

- DMF (Deep Matrix Factorization) [56]. Similar to BPMF, clinical trial records are converted into drug-disease matrix. Differently, it leverages deep structure learning model to represent drug and disease. Following [56], rows and columns of drug-disease matrix are believed to contain rich structured knowledge and are used as the input feature for neural network. For both drug and disease, the neural architecture is three-layer feedforward neural network, the latent dimensions of three layers are 500, 200, 100, respectively. - BDMF (Bayesian Deep Matrix Factorization) [53]. It extends DMF in probabilistic version and consider uncertainty estimation to enhance the performance. The setup is same as DMF. The difference is that the output of neural network becomes the mean and log-variance of the embedding vector.

- D-Embed (Dynamic Drug-Disease Embedding) is adapted from dynamic word embedding [4]. It models drug molecules and diseases' evolution over time using latent trajectories in an embedding space. The decay rate of moment estimate is set to 0.9 . Regarding time-evolutionary prior, the global diffusion constant is set to 1 . Variational inference is used to infer the embedding vector. - DeepDDI is adapted from [40]. It uses convolutional neural network (CNN) and Graph Attention based model (GRAM) [15] to encode drug molecule and ICD-code, respectively. CNN learns molecule representation from SMILES strings. Concretely, SMILES string are fed into three consecutive 1D-convolutional layers with increasing number of filters. The numbers of filter for three convolutional layers are set to $32,64,96$, respectively. The kernel sizes are set to $4,6,8$, respectively. Then it is followed by a max-pooling layer and a fully-connected networks. The attention weights in GRAM are estimated by a two-layer neural network with hidden dimension 20 and 1, the output scalars are normalized by softmax function. Then the concatenation of drug and disease representations are fed into several fully-connected layers and make binary prediction.

- GraphDDI is adapted from [13, 38]. It uses graph convolutional network (GCN) [28] to represent drug molecule and Graph Attention based model (GRAM) [15] to represent ICD-code. The hidden dimensions of GCN and GRAM are both 100. The depth of GCN is 4. Average function is leveraged as the GCN's readout function. The hyperparameter of GRAM is the same as DeepDDI. Then, same with DeepDDI, it feeds the concatenation of two representations to a fully-connected network and predict the binary label. 